lated one prospective confinement case after she had been exposed to influenza and she developed a severe attack of pneumonia, but recovered without having a miscarriage.

It will-be seen that there is practically a unanimity of opinion in favor of the idea that active immunization by the use of vaccines may be obtained, a fact which while not, of course, to be considered as scientific proof, still should be considered to have distinct value. Another interesting note is found in the comment upon reactions. These, while infrequent, were almost always mistaken for the beginning of influenza, being characterized by sudden chill, fever, headache and prostration, persisting for a few hours and subsiding gradually.

It may be of further value to note in combining the above figures, excluding the Bridge-. port Board of Health and the Lovell \& Covell Company, where comparisons are indefinite, that there was disease incidence among those inoculated of approximately $3.5 \%$, while at the sarne time among others not inoculated the disease incidence amounted to $28 \%$ and under similar surroundings. Among those immunized the incidence of pneumonia was very low, there being only fifteen cases definitely reported. The total of eight deaths has been reported, these all coming from the Allentown State Hospital where three of the patients had received but one inoculation and three, two inoculations. Apart from this the mortality records are clear.

In preparing this paper, the writer fully realizes the fact that epidemics afflict first those most susceptible and that measures for immunization employed later upon those not yet infected may consist of administering them to persons already naturally immune. As such, it is obviously unfair to compare early morbidity among a non-immunized community and later morbidity among a hopefully partially immunized one after the most susceptible have already become diseased.

In the Allentown report, however, this possibility seems to be successfully met when it is noted that of those vaccinated persons contracting the disease $36 \%$ did so within one week of the first inoculation when the immunity should be theoretically only beginning and $57 \%$ did so after the fourth week when the immunity was decreasing. It would suggest that for a period of three or four weeks, a distinct degree of resistance to infection might be produced.
Again, in the report of Dr. Phillips, no inoculated nurses in the hospital with which he is associated contracted the disease until after three weeks subsequent to inoculation, even though constantly exposed.

Dr. Leard also reports occurrence of the disease in vaccinated cases later than four weeks after inoculation.

These notes seem strongly to suggest that there is a period of three to five weeks subsequent to inoculation during which a distinct degree of immunity exists.

With these facts in mind and even with the incomplete returns necessarily incident to an almost unprecedented epidemic at a time when the medical profession of the country was seriously depleted by war, it has seemed that the results reported in this article deserve to be thus recorded.

\section{PRIMARY SARCOMA OF THE STOMACH.} REVIEW OF THE CASE SIX YEARS: AFTER OPERATION.

\section{BY A. R. Kimpton, M.D., F.A.C.S., Boston.}

In .June, 1914, in this JournaL, ${ }^{1}$ the case of round celled sarcoma of the stomach which is here to be reviewed was reported. Coincidently Frazier. ${ }^{2}$ published an article on gastric sarcoma in which he states that of 12 cases of sarcoma of the stomach the longest period of survival after operation was 14 years, while the remaining" cases survived " 2 two years, 1 one vear, 1 had recurrence in three years, and $\delta$ were reported as well from two to eleven months after operation."

The following case is of particular interest in that the patient is alive and perfectly well, with no evidence of return of the sarcoma five years and ten months after an extensive resection of the stomach for a very rapidly growing sarcoma of the round celled type. She is able to drive, oil and grease, and even change the tires of her own car.

(Quotation from Journal of June 11, 1914.) $^{1}$

"The following case is of interest, not only because of the comparative rarity of gastric sarcoma, but also because of the fact that the patient was known to have had an abdominal tumor for years. Yet at the time of operation she was not anemic or emaciated, nor did she have any gastric symptoms other than indigestion (apparently hyperacidity). 
History. Physician's wife, 30 years of age. F'ather and mother living and well. Remainder of family well. As a small girl from three to five years of age, she was subject to severe attacks of pain in epigastrium and other regions of the abdomen. These attacks lasted until relieved by drugs, and were followed by great weakness lasting three or four days. The physicians called were unable to assign any cause. On one occasion ether was administered to relieve the patient. Menses began at eleven years of age. As a young girl she was troubled a great deal with "sour stomach," and at the time of her marriage was in the habit of taking a large amount of sodium bicarbonate, which she gradually ceased to do, and for eighteen months had no trouble at all with her stomach.

Patient states that she discovered the tumor when eleven years old, but never spoke of it until after marriage, four years ago. It has troubled her more or less by occasionally being sore and painful. Within the last two years the tumor has increased in size gradually and has given more discomfort in every way. The digestive upsets have been more frequent, at times necessitating only liquid diet, but the patient never vomited.

With the exception of these attacks of "indigestion" the patient considered herself in perfect health and certainly appeared perfectly well.

Operation. December 29, 1913. Abdomen opened through an incision made below the umbilicus. Pelvic contents were found to be normal and a movable mass was found in the upper abdomen, which could be pulled down as far as the umbilicus. There were no adhesions. Extending the incision to a little above the umbilicus, it was found that a tumor of the stomach was to be dealt with. There was not only the large tumor, but many smaller ones. . . . The tumor mass was local, so far as conld be made out, although there were palpable somewhat enlarged lumbar nodes. A partial resection of the stomach was decided upon. It was necessary to resect so low on the duodenum that the stump was turned in with considerable difficulty. The stomach, likewise, had to be resected high in order to take in all of the tumor. The resection was done with an actual cautery. The lines of suture were reinforced with omentum so far as possible. The remaining portion of the stomach was so small that a posterior gastro-enterostomy was accomplished with some difficulty. There were some enlarged nodes along the greater curvature of the stomach which were removed with the tumor. A cigarette drain was left in the upper abdomen and the abdomen closed in layers. The patient went back to bed in excellent condition with a pulse of 110 . The drain was removed on the second day and was not replaced. The convalescence was uneventful throughout.

The patient left the hospital on the twentyfirst day, having lost only three pounds in weight, and since then has been in perfect health. A bismuth x-ray was taken six weeks after the operation by Dr. George, as shown by the illustration. The specimen (see illustration) was examined by Dr. William F. Whitney, and his report is as follows:

Microscopic Examination showed a solid grcwth of irregularly rounded cells separated into small lobules by connective tissue bundles, and with but little intercellular substance between the cells. In many of the cells the nuclei were eccentric. Here and there were mitotic figures and double nuclei were occasionally seen.

Diagnosis. Round cell sarcoma. The small tumors were examined as well as the larger. As the drawing shows, the tumors varied in size, some being pedunculated; were largely at the pyloric end of the stomach and did not cause any stenosis. The largest tumor was partly broken down. This is the usual condition found in the round-celled type, which is the most common type of gastric sarcoma."

A second bismuth x-ray was taken April 24, 1918 , four years and four months after operation (see illustration). It shows no indication of recurrence of the sarcoma.

Although primary sarcoma of the stomach is not the rarity it was once thought to be, it is still uncommon enough ${ }^{3}$ to warrant careful study of such cases. The proportion of sarcoma, among malignancies of the stomach, is usually stated now as low as two per cent., ${ }^{4}$ but might be higher were it not that sarcoma of the stomach is frequently mistaken for carcinoma ${ }^{2}$ and so is recorded erroneously.

The character of the sarcoma that was found in this case would lead us to expect an early recurrence, because the small round-celled type is the one most likely to metastasize. Warner 

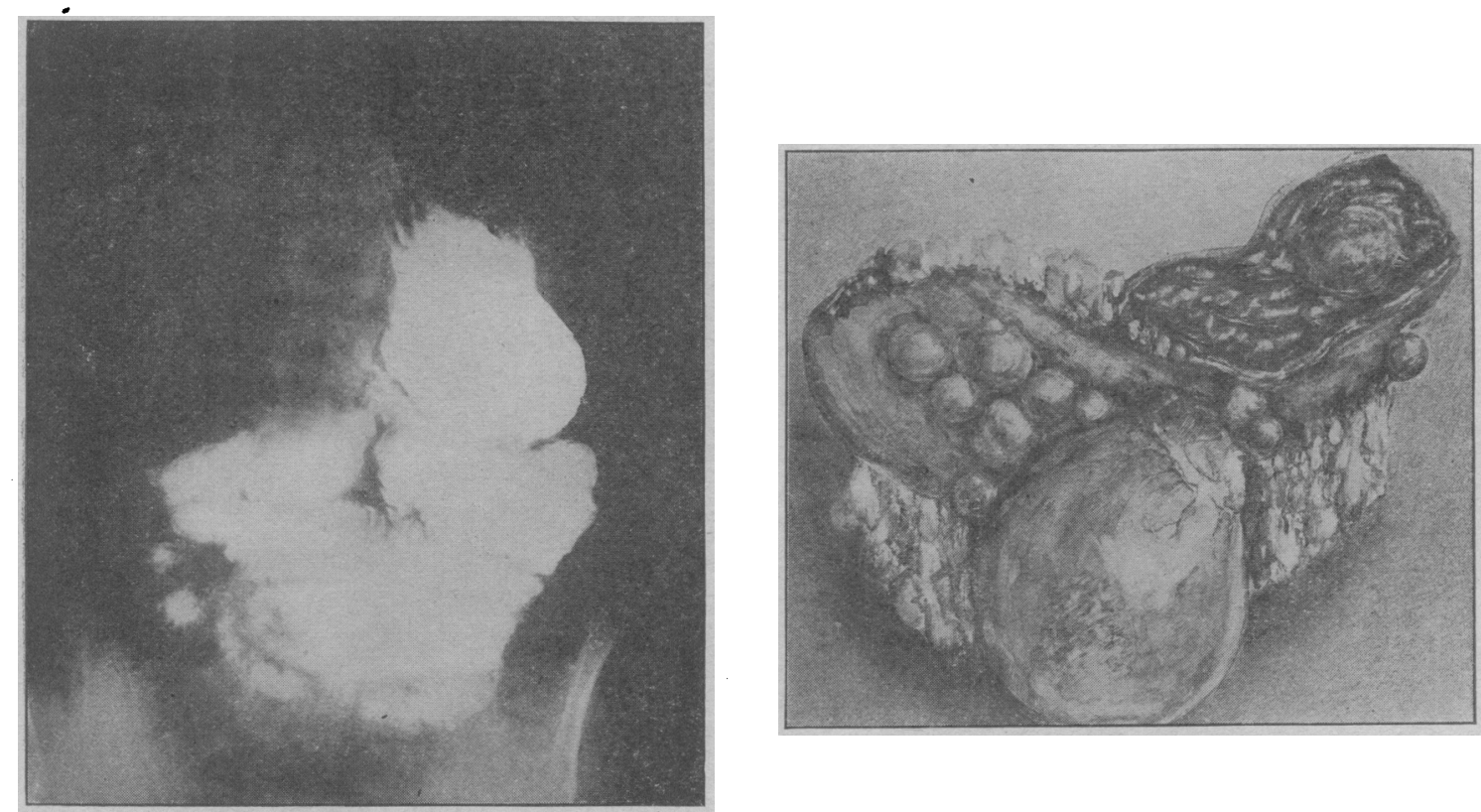

A. II: Geroma

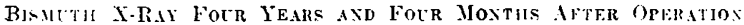

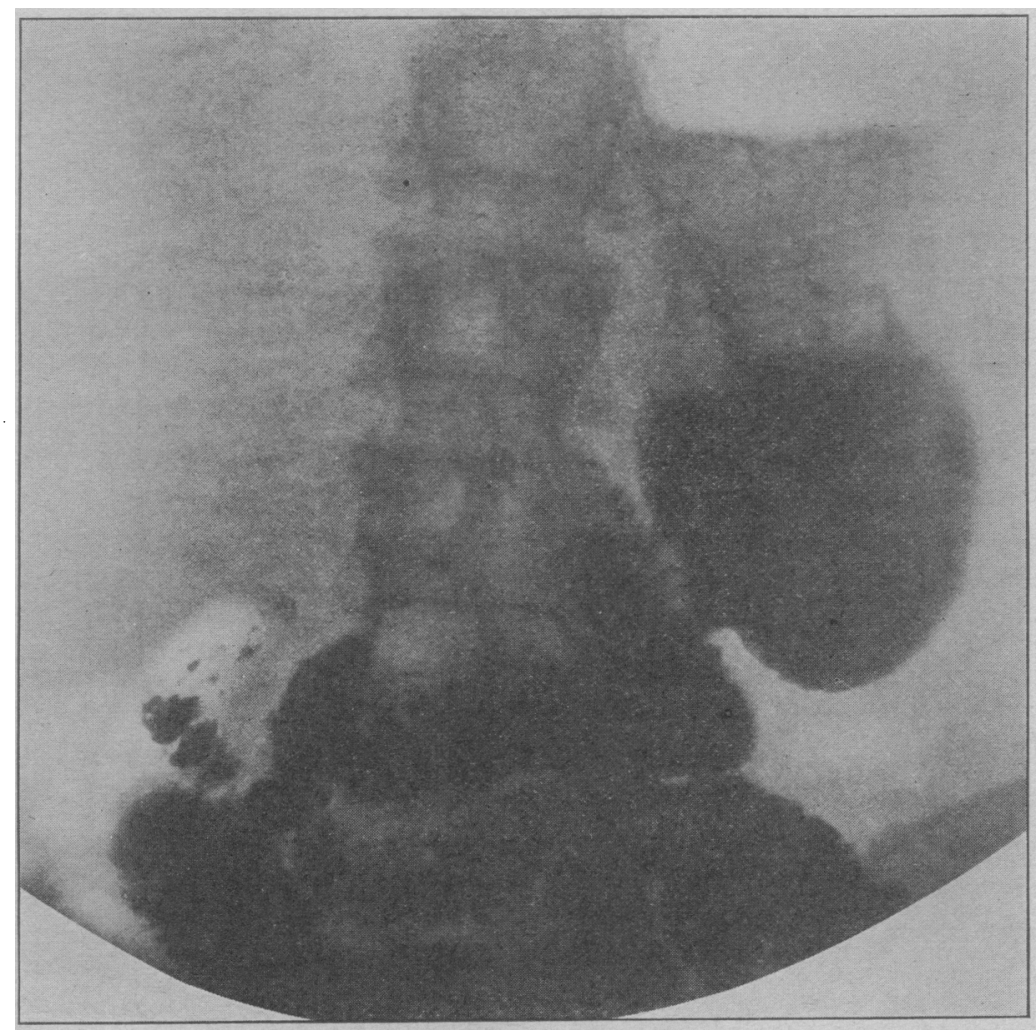

A. W. George

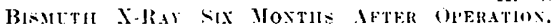

has divided sarcoma of the stomach into four Mallory; in its scirrhous form is known as groups, according to their origin. The true Ifodglin's disease), and endothelioma from the fibro-sarcoma arises from connective tissue, the endothelial cells of the blood vessels. By some leiomyo-sarcoma from smooth muscle cells of writers endothelium is not classified as a form the wall of the stomach, the lympho-sarcoma of epithelium, which accounts for the listing from the lymphoid-nodules (this, according to of the tumors of this tissue under sarcoma. It 
will be remembered that a sarcoma is a nonepithelial malignant tumor. ${ }^{5}$

Warner says that leiomyo-sarcoma is the slowest in growth, and least likely to metastasize. Lympho-sarcoma is the form of gastric sarcoma which most frequently results in metastasis. Any round celled sarcoma may look like lympho-sarcoma, but on more careful examination with differential stains it may prove to be derived from smooth muscle or from fibrablasts. In other words, this tumor, although round celled, might belong to the leiomyo-sarcomatous group. If so its failure to reappear might be expected, were it not for the fact that the very rapidity of their growth would explain the presence of round cells in the neoplasm. The round cells found in this sarcoma if not primarily lymphoid cells of the more malignant lympho-sarcoma, are to be accounted for because they have increased so rapidly that they have had no opportunity for development into the spindle form of cell that is typical of the leiomyo-sarcoma when it is less readily metastatic.

Since it is known that an abdominal tumor had existed in this patient for years it is possible that the tumor was originally a leiomyoma that has taken on malignant properties. Whether this ease, then, is a rapidly growing form of leiomyo-sarcoma or a lympho-sarcoma, a recurrence might have been expected earlier than five years after operation. It is to be hoped that, nine years later, this case will be alive to be reported as surpassing the record that Frazier has given of 14 years' survival after operation for sarcoma of the stomach.

\section{REFERENCES.}

1 Primary Sarcoma of the Stomach, A. R. Kimpton, Boston MrdiCAL AND SURGICAL JOURNAL, Vol. clxx, No. 24 pp. 915, 916, June 11, 1914.

2 Sarcoma of the Stomach, C H. Frazier, Am. Jour. Med. Sci.,

Sarcoma of the Stomach, C H. Frazier, Am. Jour. Med. Sci.,
Vol. cxlvii, No. 6, p. 781, June, 1914.

${ }^{3}$ Sarcoma of the Stomach, L. E. Barrington-Ward and E. H. Shaw,

Brit. Jour. Surg., Bristol, Vol. iv, pp. 301-303, 1916.

4 Surgery of the Stomach, B. Lewisohn, Internat. Abstract of

Surg., pp. 193-210, March, 1919.

"Sarcoma of the Stomach, Frank Warner, Ohio Med, Jomr., Col-

umbus, Vol. xiii, pp. 647-649, 1917.

Sarcoma, E. E. Tyzzer, Ref. Hand Book of Med. Soc. N. Y., Vol. vii, pp. 639.649, 1917 .

Destruction of Mrdical Records by Fire. -A fire which destroyed McCoy Hall and other buildings of the Johns Hopkins University on November 27 resulted in the loss of valuable libraries and records of the school of hygiene and public health, which occupied the second floor of MeCoy Hall.

\section{FUNCTIONAL HEART DISTURBANCES IN WOMEN.*}

By Florence L. Meredith, M.D., New York,

Consultant, United States Public Health Service; Director, New York Health Gentre for Women and Girls; Medical Examiner, Wellesley College; Instructor in Surgery, Tufts College Medical School.

Is the physical examinations conducted at the above clinic and at a large factory and a girls' college in Boston, the conclusion has been reached that a very much larger number of girls and women between the ages of 16 and 30 show signs of cardiac feebleness than would be supposed. Myocardial sub-development, a term which seems to describe it best, gives physical signs which have often caused these cases to be accounted for on the grounds of valvular disease, myocarditis, faulty innervation or anemia. Many of these cases have been called "nervous heart," in which the nerves were undoubtedly doing the best they could with the poor material in the heart muscle upon which to work. Frequently they have been called "weak" heart, without indicating to the patient that the heart muscle is only functionally weak, and not weak from disease, and without associating the weak heart with the general weak musculature throughout the body. Many cases in which the two were associated, and both attributable to lack of suitable bodily activity, have had treatment prescribed based on súch a diagnosis, and have responded in such a way as to confirm the diagnosis.

Data are available on 2,000 examinations, in about 800 of which under-development of the myocardium was found. The examinations were taken for determining fitness for admission into college, fitness for work in industrial and other lines, fitness for gymnasium work, and for general prophylactic purposes. For the most part the girls examined belonged to the working class. Out of the given 2,000, 1,500 were factory or mill operatives, 300 being store or office clerks, the remaining 200 being students, teachers, home workers and the like.

It was found that out of these $2,000,800$, or $40 \%$, had feeble but otherwise normal hearts; 200 , or $10 \%$, were considered probably, or defi-

- Address given on September 15, 1919, before a national conference of women doctors in New York. 